\title{
Circulating Cytokine Profiles of Polyparasitized Individuals in Gabon.
}

Noé Patrick M'Bondoukwé ( $\square$ mbondoukwenoe@gmail.com)

Department of Parasitology-Mycology of Tropical Medecine of the Faculty of Medecine of the Université des Sciences de la Santé https://orcid.org/00000002-4994-3355

Reinne Moutongo

Department of Parasitology-Mycology of Tropical Medicine of the Faculty of Medicine of the Université des Sciences de la Santé

Komi Gbédandé

Centre d'Etude et de Recherche sur le Paludisme Associé à la Grossesse et à l'Enfance

Jacques Mari Ndong Ngomo

Department of Parasitology-Mycology of Tropical Medicine of the Faculty of Medicine of the Université des Sciences de la Santé

Tatiana Hountohotegbe

Centre d'Etude et de Recherche sur le Paludisme Associé à la Grossesse et à l'Enfance

Rafiou Adamou

Centre d'Etude et de Recherche sur le Paludisme Associé à la Grossesse et à l'Enfance

Jeanne Vanessa Koumba Lengongo

Department of Parasitology-Mycology of Tropical Medicine of the Faculty of Medicine of the Université des Sciences de la Santé

Kowir Pambo Bello

Ecole Normale Supérieure

Denise Patricia Mawili-Mboumba

Department of Parasitology-Mycology of Tropical Medicine of the Faculty of Medicine of the Université des Sciences de la Santé Adrian John Frederick Luty

Centre d'Etude et de Recherche sur la Paludisme Associé à la Grossesse et à l'Enfance

Marielle Karine Bouyou-Akotet

Department of Parasitology-Mycology of Tropical Medicine of the Fcaulty of Medicine of the Université des Sciences de la Santé

\section{Research}

Keywords: malaria, helminths, intestinal protozoa, coinfection, Th1/Th2 balance

Posted Date: September 10th, 2020

DOI: https://doi.org/10.21203/rs.3.rs-24917/v2

License: (c) (i) This work is licensed under a Creative Commons Attribution 4.0 International License. Read Full License 


\section{Abstract}

Background: Malaria, blood-borne filarial worms and intestinal parasites are all endemic in Gabon. This geographical co-distribution leads to polyparasitism and consequently, the possibility of immune-mediated interactions between different parasite species. Intestinal protozoa and helminths could modulate antimalarial immunity, for example, thereby potentially increasing or reducing susceptibility to malaria.

Methods: Blood and stool samples were collected during cross-sectional surveys in five provinces of Gabon. Parasitological diagnosis was performed to detect plasmodial parasites, Loa loa, Mansonella perstans, intestinal helminths (STH) and protozoan parasites. Nested PCR was used to detect submicroscopic plasmodial infection in individuals with negative blood smears. Cytometric Bead Array was used to quatify interleukin (IL)-6, IL-10 and Tumor Necrosis Factor (TNF)-a in plasma of subjects with different parasitological profiles.

Results: Median IL-6 and IL-10 levels and the median IL-10/TNF- a ration were all significantly higher among individuals with Plasmodium (P.) falciparum infection compared to other groups $(P \leq 0.0001)$. The median TNF-a level and IL-10/IL-6 ratio were higher in subjects with STH $(P=0.09)$ and $P$. falciparum -intestinal protozoa co-infection ( $P=0.04)$, respectively. IL-6 $(r=-0.37 ; P \leq 0.01)$ and IL-10 ( $r=-0.37 ; P \leq 0.01)$ levels, and the IL-10/TNF-a ratio $(r=-0.37 ; P \leq 0.01)$ correlated negatively with age. Among children under five years old, the IL-10/TNF- $a$ and IL-10/IL- 6 ratios were higher in those with intestinal protozoan infections compared to uninfected children. The IL-10/TNF-a ratio was also higher in children aged 5-15 years and in adults harbouring blood-borne filariae compared to their control counterparts, whereas the IL-10/IL-6 ratio was lower in those aged 5-15 years with filariae and intestinal parasites but higher in adults with intestinal parasitic infections.

Conclusion: Asymptomatic malaria is associated with a strong polarization towards a regulatory immune response, presenting high circulating levels of IL-10. P. falciparum/intestinal protozoa co-infections are associated with an enhanced IL-10 response. Immunity against malaria could differ according to age and carriage of other parasites.

\section{Introduction}

In Gabon, malaria and intestinal parasite infections (IPIs) as well as filariasis are frequently diagnosed in the population, with the prevalence varying from $1 \%$ to $75 \%[1,2]$. This geographical co-distribution can lead to polyparasitism with possible interactions between parasite species. Studies on the association between Plasmodium and helminths have shown that helminths could have either a protective, a detrimental or a neutral effect on plasmodial infections. For example in Mali, Schistosoma (S.) haematobium delayed the appearance of clinical malaria in children, while in Gabon and in Senegal it was reported that Ascaris lumbricoides and S. haematobium increased the risk of clinical episodes [3-5]. Shapiro and colleagues found no interactions between these STH and malaria parasites [6]. Although the epidemiological patterns and/or the clinical consequences of polyparasitism are being increasingly studied, data concerning the immune responses and the susceptibility to other diseases of individuals exposed to or infected by different helminth or protozoal parasites in endemic areas remain scarce. Relevant information for Gabon does not exist. Understanding the immune response elicited by each pathogen in the case of co-infection could help with the management or the prevention of the deleterious effects of polyparasitism.

Levels of pro- and anti-inflammatory cytokines that are influenced either by the environment or by other parasites can reflect $P$. falciparum induced immunity and morbidity of populations living in regions with different malaria endemicity. Indeed, interleukin (IL-) 10 was shown to be a predictor of the occurrence clinical malaria in highly endemic areas of India while IL-6, IL-10 and IL-12 were associated with disease outcome in a non-endemic region [7]. With respect to Plasmodium-helminth co-infections, helminths orient the immune response toward an anti-inflammatory pathway whilst plasmodial infection is known to elicit a strong pro-inflammatory response [8,9]; filaria-helminth co-infection is associated with a low IL-10 level whereas that of IL-6 was described to be lower in the case of Plasmodium-hookworm co-infection [10].

On the other hand, chronic intestinal helminthic and/or filarial infection are associated with high levels of IL-10 [11]. IL-10 downregulates the functions of immune cells that release pro-inflammatory cytokines, both preventing the elimination of worms and protecting the host against helminth infection-related symptoms. IL- 6 is also described in the literature to increase significantly among populations infected with helminths [12]. However, the level of TNF- $a$ is reported not to vary [12]. Thus, intestinal parasite and filarial carriage could influence the susceptibility to and/or the course of malaria which is known to differ between urban and rural settings in high transmission areas. As an example, in a rural setting, anti-malarial immunity was shown to be modulated by infection with Trichuris trichiura [13,14]. Nevertheless, in urban and suburban areas data on such interactions are lacking. The heterogeneity of Plasmodium spp. Prevalence observed in Gabon can be due to different susceptibility patterns to $P$. falciparum infection according to the presence and type of other parasitoses. The study presented here was conducted in areas of Gabon not yet investigated that have different levels of malaria endemicity. In the country, among intestinal parasitism, protozoan predominate in single or in co-infection with $P$. falciparum compared to STHs [15,16], but the interaction between such parasite species carriage, with $P$. falciparum-related immunity has not been studied, as non-pathogenic protozoan carriage does not constitute a public health problem. However, experimental studies on Entamoeba (E.) histolytica and Giardia (G.) intestinalis, have demonstrated that these parasite carriage is associated with the production of pro-inflammatory cytokines $[17,18]$. Subtypes of Blastocystis spp, those prevalence is increasing in the country, are now considered as pathogenic intestinal protozoa. And In humans, this parasite was also associated with an immunomodulation, more precisely by downregulating the immune response towards another antigen as helminths $[18,19]$. This study evaluated the impact of intestinal parasite (helminths or protozoa) and blood filaria carriage on the level of cytokines involved in malaria immunity during coinfection with helminths and protozoa in urban and rural areas of Gabon.

\section{Methods}

\section{Study sites and populations}


This study was nested in cross-sectional surveys on asymptomatic malaria carried out from September 2013 to June 2016 in five out the nine provinces of Gabon with different degrees of urbanization (Figure 1). Samples taken from individuals participating in villages located around the main towns of the Ogooué-Ivindo and Haut-Ogooué provinces, and those from patients at the Centre Hospitalier Régional (CHR) d'Oyem, (Woleu-Ntem province), the CHR de Koula-Moutou and Dienga (Ogooué-lolo province), the CHR de Melen and the Lalala Public Health Center (Estuaire province) were used for this study. Lalala is a suburban area of the capital city of Libreville, an urban area; the CHR of Melen is located in a suburban area and the CHR d'Oyem, of Koula-Moutou as well as villages in the Ogooué-lvindo and Haut-Ogooué and Dienga are located in rural areas.

Inclusion criteria for sample selection included for the study participants: absence of fever (axillary temperature $\leq 37.5^{\circ} \mathrm{C}$ ) or absence of history of fever the day of the screening and during the week preceding the consultation, absence of other clinical symptoms suggestive of malaria, absence of antimalarial drug uptake the last two weeks, absence of any other severe medical condition and sickle cell disease, permanent residence in the study area, agreement to fill the questionnaire and written informed consent.

All the plasma controls were those from individuals with no history of or current $P$. falciparum or intestinal parasite infection.

\section{Procedures for sample selection for the immunological analysis}

During the main surveys, patient recruitment, demographic, socioeconomic, environmental and parasitological data were collected in standardized case report forms. Among the 843 patients recruited for the main surveys, 400 provided blood and stool samples. After the microscopic diagnosis of blood and intestinal parasites, blood samples of those with negative blood smears were screened for submicroscopic plasmodial infection by nested polymerase chain reaction after DNA extraction using QIAamp® kit.

Then, five groups of samples from participants with different profiles of parasitic infection were constituted: plasma from those with (i) Plasmodium spp. infection alone, (ii) helminth infection (blood-borne filariae and intestinal worms), (iii) intestinal protozoan infection alone, (iv) Plasmodium spp./helminth coinfection and (v) Plasmodium spp./intestinal protozoan co-infection. The latter group included samples without detected parasites according to the method used for diagnosis; individuals with no parasites constituted the control group.

\section{Ethical consideration}

This nested study received ethical clearance from "Comité National d'Ethique pour la Recherche" (CNER) of Gabon. The protocol and the questionnaire were also approved by the Ministry of Health.

\section{Sample processing}

After collection, whole blood was used for microscopic malaria and blood filarial diagnosis. After centrifugation blood pellets were stored at $-20^{\circ} \mathrm{C}$ for the molecular detection of $P$. falciparum; plasmas were directly stored at $-20^{\circ} \mathrm{C}$ on site, then transported to the laboratory in a cooled container and stored at - $80^{\circ} \mathrm{C}$ prior to immunological analysis. The participants were provided with clean, labelled stool collection pots with clear instructions to ensure that stool samples were collected correctly.

\section{Parasites microscopic diagnosis}

The detection of plasmodial parasites was performed by thin and thick smears using the Lambaréné method [20]. Whole blood direct microscopic examination and leukoconcentration techniques allowed for the identification of blood filariae L. loa and M. perstans [21]. To determine the presence of intestinal parasites, three techniques were performed: direct stool examination, Merthiolate-lodine-Formaldehyde coloration and parasite culture. These techniques are described in detail elsewhere [15].

For all the techniques for the detection of blood and intestinal parasites, two trained operators were necessary to validate a result. In case of discordance, a third reading was carried out. This procedure is carried out during routine examinations by the technicians of the laboratory of Parasitology.

\section{DNA extraction and submicroscopic plasmodial infection}

DNA was extracted from peripheral blood collected in EDTA tubes using the QIAamp® kit (QIAGEN®) according to the manufacturer's instructions. The 18S rRNA malarial genes were amplified by nested PCR according to the protocol described by Snounou and Singh [22]. Briefly, for the first reaction, rPLU 1 ( 5 ' TCA AAG ATT AAG CCA TGC AAG TGA 3') and 5 ( $5^{\prime}$ CCT GTT GTT GCC TTA AAC TCC $3^{\prime}$ ) primers pairs were used. The product generated in this reaction served as a template in the second reaction, performed with an rPLU 3 (5' TTT TTA TAA GGA TAA CTA CGG AAA AGC TGT 3') and 4 (5' TAC CCG TCA TAG CCA TGT TAG GCC AAT ACC $3^{\prime}$ ) primers pairs allowing the generation of a $235 \mathrm{bp}$ fragment. The visualization of the PCR products was carried out using $2 \%$ agarose gel 
electrophoresis. At each experiment, positive and negative controls were included to validate the obtained results.. Participants identified as Plasmodium sp. infected had positive PCR results or positive thick smears.

\section{Circulating cytokines measurement}

A Cytometric Bead Array human cytokine kit from Becton Dickinson (CBA kit, BD Biosciences, San Diego, CA, USA) was used to measure plasma levels of IL-6, IL-10 and TNF-a according to the manufacturer's instructions. Samples were centrifuged and one volume of the supernatant obtained was diluted into two of the assay diluent. Then, the technique was realized as described by Böstrom and colleagues [23]. The highest standard concentration was $2500 \mathrm{pg} / \mathrm{mL}$ and the lowest was $5 \mathrm{pg} / \mathrm{mL}$. The calibration, the sample acquisition in duplicate and the standard was performed using a BD FACSCalibur flow cytometer (FACSCalibur, Becton Dickinson, Le pont de Claix Cedex, France), and results were analysed with FCAPArray v1.0.1 software (SoftFlow, Pécs, Hungary). The detection limits of cytokines were $1.6 \mathrm{pg} / \mathrm{mL}, 0.13 \mathrm{pg} / \mathrm{mL}$ and $1.2 \mathrm{pg} / \mathrm{mL}$ for, respectively, IL- 6 , IL- 10 and TNF-a. If the cytokine concentration was below the detection limit, a value corresponding to half of the detection limit was assigned to the sample.

\section{Statistical analysis}

Statview Version 5.0 (SAS Institute Inc.) was used for statistical analysis and GraphPad Prism Version 5.03 and Statview Version 5.0 (SAS Institute Inc.) for graphical representations of box plot and scatter column. Cytokine concentrations were transformed to $\log _{10}$ function for graphical representation. IL-10/TNF$a$ and IL-10/IL-6 ratios were calculated for each sample. The ranges of cytokine concentrations, Plasmodium sp., L. Ioa and M. perstans parasitaemia and eosinophilia did not follow normal distributions and descriptive analyses of these quantitative variables are presented with medians [interquartile ranges, IQR: $25^{\text {th }}-75^{\text {th }}$ quartiles]. Tests for comparison of cytokines and ratios were performed firstly using the Kruskal-Wallis test for a global comparison, then the Mann-Whitney U test for a comparison of medians of two groups with different infection profiles. Only the Mann-Whitney test for pairwise comparisons with Bonferroni correction was used to determine the significant difference in the cytokine concentrations and cytokine ratio. In order to perform multivariate analysis, R software was used to build and evaluate some models. F-tests were performed to statistically test the equality of means into different groups, according to age, location, parasitaemia. A $p$-value less than 0.05 was considered significant. Then, a correction Bonferroni test was used for the pairwise comparison of cytokines concentrations adjusted for age group [24]. In this case, a $p$-value of less than $0.0033(0.05 / 15)$ was considered significant. Pearson's $r$ test was used to evaluate the correlation between the cytokines level/cytokine ratios (variable $Y$ ) and age (variable $\mathrm{X}$ ). Due to the difference of immune response between populations according to urbanization and to avoid confounding factors, areas were split in urban and rural areas and correlation coefficients values were obtained for each area.

\section{Results}

\section{Patients and parasites}

Samples of 240 participants were selected for the immunological analysis (Figure 2). Out of the 208 patients for whom the area was known, 172 ( $82.7 \%$ ) were from a rural area (Table 1). Age was recorded for 224 of them and the median age was 22.5 [6.0 - 48.8] years old. Adults represented more than half of the study population $(58.0 \% ; 130 / 224)$ and children less than 5 years old, $21.0 \%(47 / 224)$. The sex ratio (number of male/number of female) was 0.83 (109/131) and did not differ according to age and site.

Microscopic and molecular diagnoses combined showed the prevalence of single Plasmodium infection to be 20.8\% (50/240). P. falciparum was the only species identified. Intestinal helminths (STH) and filariasis infections were detected in $43(17.9 \%)$ and 48 (20.0\%) individuals respectively, and intestinal protozoan monoinfection in 39 (16.3\%). A. lumbricoides (14.6\%; 35/240), T. trichiura (13.7\%; 33/240), B. hominis (36.2\%; 87/240), E. histolytica/dispar (7.1\%; $17 / 240$ ) were the most frequently detected intestinal parasites; $G$. duodenalis and $S$. stercoralis were each one found in $5 / 240(2.1 \%)$ samples only. Schistosoma species were not detected in any of the samples. The rates of $P$. falciparum/STH and $P$. falciparum/filariae co-infections were $2.9 \%$ ( $7 / 240)$ and that of $P$. falciparum/intestinal protozoan co-infection, 7.1\% (17/240) (Table 1).

Prevalence of parasitic infection of any type was highest in adults $(87.7 \% ; 114 / 130)$, followed by those aged 5 to 15 years old (87.2\%; $41 / 47)$ and lowest in children less than 5 years old $(72.3 \% ; 34 / 47)\left(\chi^{2}=33.932, d f=2, P<0.0001\right)$. Adults were more frequently infected than children (Table 1$)$. The prevalence of filariae (97.7\%), STH (70.5\%) and intestinal protozoa (48.7\%) was significantly higher among adults compared to children $\left(\chi^{2}=71.747, d f=14, P<0.0001\right)$ (Table 1). Moreover all those co-infected with $P$. falciparum/filariae were adults. The proportions of individuals with $P$. falciparum/STH co-infections and $P$. falciparum/intestinal protozoan co-infections were greatest respectively in younger children (50.0\%) and those aged between 5 and 15 years old (52.9\%).

P-values presented in this paragraph are considered globally for comparison of the parasitaemia and filaremia between groups. To explore more in details the significance, see the Additional file 1: Table S1. The median $P$. falciparum parasitaemia was significantly higher in cases of monoinfection (9450 [1296-36400] $\mathrm{T} / \mu \mathrm{L}(P \leq 0.04)$ while it was lower in those with either STH (42 [12.3-681.5] T/ $\mu \mathrm{L})$, filarial (350 [16-1400] T/ $\mu \mathrm{L})$ or intestinal protozoan $(749[35-10150] \mathrm{T} / \mu \mathrm{L})$ co-infections $(P \leq 0.04)$. L. loa and $M$. perstans parasite densities were comparable between groups. Intestinal protozoa were found to be more frequently associated with other parasites $(28.7 \% ; 69 / 240)$. 
IL-6, IL-10 and TNF-a median levels were 14.2 [4.7-68.0] pg/mL, 11.1 [8.0-15.2] pg/mL and 5.4 [4.6-6.8] pg/mL respectively. The IL-10/TNF-a ratio was 2.0 [1.43.2] and that of IL-10/IL-6, 1.2 [0.2-2.3]. The "No parasite" group was used as reference to estimate an increasing, a decreasing and comparable cytokine levels and cytokine ratios (Table 2).

\section{Cytokine levels in mono- and polyparasitized individuals}

The applied Kruskal-Wallis test showed trends towards differences $(p<0.05)$ in the cytokine rate and cytokine ratios except for TNF- $a(p=0.09)($ Table 2$)$. The descriptive analysis of the cytokine measurements showed the following results. IL-6 median levels were higher among those with single $P$. falciparum (124.5 [36.9-433.9] pg/mL) and a P. falciparum/STH co-infection (25.5 [4.4-60.8] pg/mL). IL-10 was also higher in participants with $P$. falciparum single infection (224.5 [78.0-657.9] pg/mL) and in co-infected $P$. falciparum-filariae (18.1 [12.8-125.2] pg/mL), STH (39.5 [33.0-68.0] pg/mL) and intestinal protozoa infected ones $(26.9$ [8.7-60.1] pg/mL). STH-infected individuals had a higher median TNF-a level compared to the uninfected. The IL-10/TNF-a ratio was on average 30 fold (69.9 [12.5-140.7]), 10-fold (19.7 [1.6-115.2]), 6-fold (13.0 [3.5-24.9]) and 8-fold (16.9 [2.4-86.6]) higher in those with single or $P$. falciparum co-infections with filariae, STH and intestinal protozoa respectively, compared to uninfected participants. The IL-10/IL- 6 ratio tended to be higher in those with $P$. falciparum infections alone (2.0 [0.9-3.6]), it was 2-fold (2.5 [1.8-4.7]) and 3-fold (3.6 [2.0-11.9]) higher in those with dual infections with malaria/filariae and malaria/intestinal protozoans, respectively.

Figure $4 \mathrm{a}$ and $4 \mathrm{~b}$ show pairwise comparisons of cytokine median concentrations and ratios. TNF-a levels did not differ significantly according to the presence or the type of parasitic infection (Figure 4).

After Bonferonni correction in the figure 4a and considering a $p$-value $<0.0033$, subjects with plasmodial infection only had higher level of IL-6 compared to subjects with $P$. falciparum/intestinal protozoa coinfection $(P=0.0008)$ and a trend with $P$. falciparum/STH $(P=0.0086)$. Those with STH $(8.0[4.7-12.7] \mathrm{pg} / \mathrm{mL})$ and intestinal protozoa $(8.9[6.8-14.5] \mathrm{pg} / \mathrm{mL})$ mono-infections, had lower median IL-10 levels than those with, respectively, a $P$. falciparum/STH co-infection (39.5 [33.0-68.0] pg/mL) $(P=0.0075)$ and $P$. falciparum/intestinal protozoa co-infection (26.9 [8.7-60.1] pg/mL) $(P=0.001)$. In rural area, participants with plasmodial infection only had IL-6 rate higher comparatively to patients with $P$. falciparum/filariasis $(P=0.0051)$ and $P$. falciparum/intestinal protozoa coinfections $(P=0.0049)$. Higher IL-10 concentrations were found in plasmodial-infected subjects and with filariasis compared to those with $P$. falciparum/intestinal protozoa coinfection $(P=0.0007)$ and filariasis only $(P=0.0011)$ respectively. The IL-10/TNF-a ratio was higher among patients with $P$. falciparum/filariae, $P$. falciparum/STH and $P$. falciparum/intestinal protozoa coinfections that those with filariae $(P=0.0013), \mathrm{STH}(P=0.0026)$ and intestinal protozoa mono-infections $(P=0.0029)$.

\section{Cytokine profiles in relation to age}

The levels of IL-6, IL-10 and the IL-10/TNF-a ratio were inversely associated with age of the study participants in the absence of parasitic infections (Figure 3 and Table 3). The same trend was observed in case of $P$. falciparum single infection (for IL-6 only), for filariasis and intestinal protozoal infection (for all) (Table 3). Uninfected children aged of 5-15 years old had the highest IL-10/IL-6 ratio (Table 3).

Overall, IL-10 and IL-10/TNF-a ratio did not significantly vary between infected and uninfected adults. The median IL-6 level was between 3 and 4 fold lower in case of a parasitic infection within the adult group, while an IPI was associated with a higher IL-10/IL-6 ratio.

Among children, a trend towards a lower TNF-a level in those aged below 5 years who had a STH carriage, and a higher level in those aged between 5-15 years old was noted. IL-10 median levels were always lower in case of STH whatever their age, whereas the level of IL-6 was reduced in individuals infected with filariae and intestinal parasitosis. This reduction was more pronounced for the youngest children with a STH.

The IL-10/TNF- $a$ and IL-10/IL-6 ratios had contrasting profiles when compared between older and younger children. Thus the IL-10/IL- 6 ratio was low among the infected older children and high among the youngest. The IL-10/TNF-a ratio was low in young children with STH and in older children with intestinal protozoa, while it was high in young children with intestinal protozoa.

This ratio was higher among participants aged of 5 to 15 years old (3.4), compared to unparasitized ones (2.3 [1.2-25.4]), and lower (1.6 [1.3-2.8]) in groups of subjects with filariasis and intestinal protozoa infection. Adults presented equally high ratio in case of filariasis (2.0 [1.1-3.4]) and low ratio in case of geohelminthiasis (1.1 [0.7-2.0]) compared to non-infected subjects (1.6 [1.3-1.9]). IL-10/IL-6 was 18 fold (7.1 [4.1-8.8]) and 6 fold (2.4 [1.0-5.4]) higher in younger children with STH and intestinal protozoan infections compared to unparasitized ones. Among older children, those with filariae (2.9), STH (2.9 [0.25.8]) and intestinal protozoa (2.0 [1.2-8.8]) presented lower IL-10/IL-6 ratios than control group (5.0 [1.3-8.6]). In adults, this ratio was found higher in case of STH (2.1 [1.1-5.5]) and intestinal protozoa (2.1 [0.7-7.9]) carriage (Table 3).

\section{Multivariate analysis}

Comparison of the IL-6, IL-10 and TNF-alpha levels, and of the IL-10/IL-6 and IL-10/TNF-alpha ratios in the 8 different parasitic groups adjusting by age, urbanization and $P$. falciparum parasitaemia showed no statistically significant differences (Bonferroni test, $P=0.6$ ). Cytokine concentrations and cytokine ratios were similar across the age groups after adjusting for parasitic infections and urbanization. 


\section{Discussion}

This study is the first to analyse the influence of single or multiple parasitic infections on the plasma cytokine profile of Gabonese individuals. The main aim was to determine the relationship between intestinal parasitoses, as well as loiasis, and the plasma concentrations of different cytokines implicated in malaria pathophysiology.

Lower parasitaemias were observed among participants carrying co-infections of either $P$. falciparum-intestinal parasites or $P$. falciparum-filariasis compared to those with only $P$. falciparum, suggesting an effect of intestinal parasites and filariae on $P$. falciparum multiplication. Lower parasite burdens, in the presence of intestinal helminth infections, have been reported elsewhere $[25,26]$. According to these authors, one explanation could be the existence of immune cross-reactivity between intestinal parasites and Plasmodium sp. Indeed, both parasites induce Th2 immune response and specific IgG3 produced as a result of intestinal parasitic infection could neutralize plasmodial parasites [27]. In the case of protozoan co-infections, no impact of intestinal protozoa on Plasmodium sp parasitaemia has been described.

P. falciparum-infected participants, who were asymptomatic when included, had higher median levels of IL-6 and IL-10 compared to the control group, but no difference in the level of TNF-a level. In the context of IL-10, this result is consistent with the findings of earlier studies [28,29]. The immune-regulatory and antiinflammatory cytokine IL-10 is known to downregulate Th1-type cytokines such as IL-6, TNF, and IL-1 [30]. Here, we found IL-10 to be implicated in the inhibition of the production of the TNF-a but not of IL-6 during plasmodial infection. It is possible that the downregulation of TNF is correlated with the absence of clinical signs. TNF-a did not vary in the control group compared to Plasmodium-infected participants with. TNF-a is the cytokine most implicated in the development of the clinical signs of malaria [31], whilst IL-10 and IL-6 are present at high levels in patients with symptomatic malaria [32-34]. The asymptomatic status of participants at the time of the study presented here could indicate the acquisition of premunition which limits the appearance of clinical symptoms, and/or that participants were sampled at an early stage of the infection, before progression toward symptomatic disease [35]. It is important to note that, in the current Gabonese context, younger children are found to be less frequently infected by malaria parasites than older children $[15,36]$ and the number of adults presenting with clinical malaria is increasing, suggesting a loss of malaria premunition. This epidemiological picture most likely primarily reflects the switch, since 2003 , to the use of artemisinin combination therapy for malaria in under-five year old children.

Previous studies conducted in Gabon showed malaria to be co-endemic with other parasitic infections that are present at a similar or higher prevalence (compared to malaria) and that can alter immunity to malaria (Akue et al., 2011; Bouyou-Akotet et al., 2015; M'Bondoukwé et al., 2016, 2018). Here, intestinal parasite infection (IPIs) and blood filariasis were associated with decreased Th1 (IL-6) and Treg (IL-10) responses. Blastocystis sp., the most prevalent intestinal parasite, have immunomodulatory effects with induction of pro-inflammatory cytokine responses [39]. The prevalence of Blastocystis has increased across the country $[15,16]$. Therefore, it was hypothesized that, together with its implication in dysbiosis, Blastocystis could affect cytokine production and influence $P$. falciparum carriage. A study in Pakistan showed that Blastocystis sp. type 1 was associated with low IL-10 production in the blood, but that in stool samples Blastocystis sp. generates an anti-inflammatory environment $[19,40]$.

The impact of other pathogenic intestinal protozoa on the cytokine profile was not investigated here because of the low number of infected patients and the absence of single E. histolytica and G. duodenalis mono-infection or co-infection with $P$. falciparum. However, during intestinal protozoa/ $P$. falciparum infection, a significant reduction of pro-inflammatory cytokines (IL-6, TNF-a) was observed, suggesting that infection with G. duodenalis, E. histolytica, or Blastocystis sp. decreases the $P$. falciparum-induced Th1 response, thereby contributing to the absence of clinical symptoms. This would also explain the difference in IL-10 levels between participants with intestinal protozoa and those from the control group. The implication of each of these three protozoan parasites as well as the different subtypes of Blastocystis $s p$. on the global and specific cytokine response merits further exploration.

Individuals with a low Th2-Treg/Th1 ratio are less susceptible to malaria but have a greater likelihood of clinical disease when infected [41,42]. Here we observed that IL-10 and IL-6 levels were 1.3 to 18.0-fold lower in those with malaria-intestinal parasite co-infections (both helminths and protozoa) (data not shown) compared to those with plasmodial mono-infections, although still higher compared to uninfected participants. A trend towards a down regulation of TNF-a was observed in co-infections with intestinal parasites. This regulatory effect on pro-inflammatory cytokine production during helminth and $P$. falciparum co-infections is well-described (Bustinduy et al., 2015, Sinha et al., 2010). Elevated IL-10 levels have been observed in patients with Plasmodiumschistosomiasis and malaria-hookworm co-infection (Bustinduy et al., 2015, Courtin et al., 2011, Diallo et al., 2004; al., 2003). The higher IL-10/TNF-a ratio, in the group of participants with Plasmodium mono-infection compared to Plasmodium/helminth co-infection suggests a possible lower risk of developing clinical signs in case of STH/P. falciparum co-infection as demonstrated by Frosch \& John (2013). Additionally, the IL-10/IL-6 ratios suggest that Plasmodium and intestinal protozoa co-infected participants would be associated with asymptomatic carriage of these parasites. However, a meta-analysis of young African children with helminth-P. falciparum co-infections concluded that they are more susceptible to $P$. falciparum infection [45]. Comparative analysis of uninfected unexposed individuals, infected asymptomatic and patients with clinical symptoms are needed. If such analysis confirms the present results, then it can be suggested that exposure to both IPI and malaria in the context of the observed increasing prevalence of intestinal parasites in the country would partly explain the increasing frequency of asymptomatic $P$. falciparum carriage observed in the country $[36,43,44]$.

IL-10/TNF-a ratios show that filariae-infected volunteers more than 5 years old have a higher risk of $P$. falciparum infection than participants infected with other parasites. But when the IL-10/TNF-a (except for STH infection) and IL-10/IL-6 ratios were analyzed, children with intestinal parasites were found to be more susceptible to $P$. falciparum infection presenting higher values. Intestinal helminths and filarial infections are associated with higher production of IL-10 and consequently with the inhibition of Th1-type cytokine production [12,46,47]. The high IL-10/TNF-a and IL-10/IL-6 ratios observed here in those with helminth co-infections are consistent with such observations.

The development of the anti-infectious immune response is promoted by repeated exposures and the chronicity or persistence of infections. The immune response was studied according to age in uninfected, mono-infected or polyparasitized participants. It differed according to age and type of parasitism. The negative correlation between cytokine levels and age is in favor with a greater stimulation in young children. They are actually more at risk of the studied 
parasites (with the exception of filariasis) and polyparasitism [15]. Although, the cross-sectional design of this study, does not allow to confirm that the measured cytokine levels were baseline. Nevertheless, the fact that participants lived in the study area since several months without significant interventions related to malaria or IPI, allow a comparison between the different groups determined according to the presence and the type of infection. Children with STH had low level of IL-10, as observed in Kenyan children; depending on their age, they may simply require a longer period of chronic infection to develop the typical Treg-related IL-10-dominated profile. [10]. The decrease in the IL-6 level in case of intestinal protozoan can be related to an immunoregulatory effect induced by these parasitosis; this is confirmed by the high IL-10/TNF-a ratio. The analysis of the IL-10/IL- 6 ratio which shows a different profile than that of each cytokine would highlight the predominance of the anti-inflammatory cytokine response in young children infected with STH and intestinal protozoa. In contrast, in adults, the comparable level between infected and uninfected ones, is probably linked to a better control of these infections through a protective and mature immune response.

The predominance of a pro-inflammatory cytokine profile in the absence of infection may be linked to the greater susceptibility of young children to severe malaria which is determined by high IL-6 and TNF response. This could partly explain the predominance of severe forms of malaria in young children, although they remain less frequently infected $[36,48]$. Indeed, the lower basal cytokine Th1 level in healthy children could indicate their greater susceptibility to plasmodial infection due to a less efficient control of Plasmodium multiplication that would quickly reach the pyrogenic threshold. In contrast, the downregulation of IL-10 observed in older children would be in favour of a less control of the parasite multiplication at the beginning of infection and therefore of a higher frequency of parasitaemia in this group compared to the young people.

The cross-sectional design is one of the main limits of this study. Also, plasma cytokines cannot be considered as being specifically induced by any given parasitic infection. Specific and more sensitive techniques such as stimulation of PBMC in vitro to assess responses to $P$. falciparum, intestinal parasites and filariae would be useful in this context. Equally, next generation sequencing would give a global picture of the immune response by measuring the transcript levels of Th1-, Th2- and Treg-related cytokines. The influence of helminths and intestinal protozoa on innate and adaptive responses to $P$. falciparum could thus be revealed. The small sample size in groups with different parasitic infections could also explain the lack of association between the cytokine levels and infection profiles in multivariate analysis. However, the present results already give a baseline estimation of the overall in vivo cytokine levels implicated in anti-parasite immunity in exposed individuals according to their age.

\section{Conclusion}

This study demonstrates the implications of blood filariae, intestinal parasites (helminths and protozoa) on the cytokine responses involved in susceptibility to infection with $P$. falciparum. STH would be protective against malaria according to the IL-10/TNF-a ratio, while with the IL-10/IL-6, intestinal protozoa may have a detrimental effect. These parasites which are co-endemic with Plasmodium falciparum in Gabon also seem to alter the susceptibility to $P$. falciparum infection according to age. Those more than 5 years old and adults seem to be more at risk of infection when co-infected with filariasis associated with a higher IL-10/TNF-a ratio, and when co-infected with intestinal parasites associated with a higher IL-10/IL-6 ratio. However, additional studies involving unexposed uninfected and asymptomatic or clinically ill participants are needed to better understand the dynamic interaction between specific host immune response and polyparasitism.

\section{Abbreviations}

A. Iumbricoides: Ascaris lumbricoides; CBA: Cytometric Bead Array; CHR: Centre Hospitalier Régional; CNER: Comité National d'Ethique pour la Recherche; DNA: deoxyribonucleic acid; E. coli: Entamoeba coli; E. histolytica/dispar: Entamoeba histolytica/dispar; EDTA: Ethylenediaminetetraacetic acid; G. intestinalis: Giardia intestinalis; Ig: Immunoglobulin; IL-: interleukin; L. Ioa: Loa loa; M. perstans: Mansonella perstans; N. americanus: Necator americanus; P. falciparum: Plasmodium falciparum; S. haematobium: Schistosoma haematobium; S. stercoralis: Strongyloides stercoralis; STH: soil-transmitted helminths; T. trichiura: Trichuris trichiura; T/ $\mu \mathrm{L}$ : trophozoite/microliter; Th: T helper; TNF: tumor necrosis factor; Treg: T regulatory.

\section{Declarations}

\section{Acknowledgements}

We would like to thank the members of the Red Cross in Libreville and the Ministry of Health for giving permission and logistical support for this study to be conducted. We are also grateful to the study participants.

\section{Declarations}

\section{Ethics approval and consent to participate}

This nested study received ethical clearance from "Comité National d'Ethique pour la Recherche" (CNER) of Gabon. Participants were informed before to enrolled in the study and accepted that plasma will used for immunological studies. The protocol and the questionnaire were also approved by the Ministry of Health.

\section{Consent for publication}


Not applicable

\section{Availability of data and materials}

The datasets used and/or analysed during the current study are available from the corresponding author on reasonable request.

\section{Competing interests}

The authors declare that they have no competing interests.

\section{Funding}

This study was funded by the Department of Parasitology-Mycology of Tropical Medicine, RELACS network and the Gabonese Red Cross for the data and sample collection on the field. CECAGADIS, a private corporate from Gabon, funded this project, for immunological analysis carried out in Benin.

\section{Authors' contributions}

MKB-A was the principal investigator and conceived the study with NPM. NPM carried out plasma aliquots and performed Cytometric Bead Array with KA, RA and TH in Benin. AFJL brought the technical platform and supervised the immunological analysis. MKB-A and NPM wrote the paper. DPM-M, JMNN and JMNN reviewed and edited the paper. The statistical analyses were carried out by MKB-A, MNP and KP and MKB-A, MNP proceeded to the interpretation of the data.

\section{References}

1. Maghendji-Nzondo S, Kouna LC, Mourembou G, Boundenga L, Imboumy-Limoukou RK, Matsiegui P-B, et al. Malaria in urban, semi-urban and rural areas of southern of Gabon: comparison of the Pfmdr1 and Pfcrt genotypes from symptomatic children. Malar J. BioMed Central; 2016;15:420.

2. Ateba-Ngoa U, Jones S, Zinsou JF, Honkpehedji YJ, Adegnika AA, Dejon Agobe JC, et al. Associations between helminth infections, Plasmodium falciparum parasite carriage and antibody responses to sexual and asexual stage malarial antigens. Am J Trop Med Hyg. 2016;95:394-400.

3. Adegnika AA, Ramharter M, Agnandji ST, Ateba-Ngoa U, Issifou S, Yazdanbahksh M, et al. Epidemiology of parasitic co-infections during pregnancy in Lambaréné, Gabon. Trop Med Int Heal. 2010;15:1204-9.

4. Lyke KE, Dicko A, Dabo A, Sangare L, Kone AK, Coulibaly D, et al. Association of Schistosoma haematobium infection with protection against acute Plasmodium falciparum malaria in Malian children. Am J Trop Med Hyg. 2005;73:1124-30.

5. Sokhna C, Le Hesran J-Y, Mbaye PA, Akiana J, Camara P, Diop M, et al. Increase of malaria attacks among children presenting concomitant infection by Schistosoma mansoni in Senegal. Malar J. 2004;3:43.

6. Shapiro AE, Tukahebwa EM, Kasten J, Clarke SE, Magnussen P, Olsen A, et al. Epidemiology of helminth infections and their relationship to clinical malaria in southwest Uganda. Trans R Soc Trop Med Hyg. 2005;99:18-24.

7. Sinha S, Qidwai T, Kanchan K, Jha GN, Anand P, Pati SS, et al. Distinct cytokine profiles define clinical immune response to falciparum malaria in regions of high or low disease transmission. Eur Cytokine Netw. 2010;21:232-40.

8. Hartgers FC, Obeng BB, Kruize YCM, Dijkhuis A, McCall M, Sauerwein RW, et al. Responses to malarial antigens are altered in helminth-infected children. J Infect Dis . 2009;199:1528-35.

9. Boef AGC, May L, van Bodegom D, van Lieshout L, Verweij JJ, Maier AB, et al. Parasitic infections and immune function: Effect of helminth infections in a malaria endemic area. Immunobiology . Elsevier GmbH.; 2013;218:706-11.

10. Bustinduy AL, Sutherland LJ, Chang-Cojulun A, Malhotra I, DuVall AS, Fairley JK, et al. Age-Stratified Profiles of Serum IL-6, IL-10, and TNF- Cytokines Among Kenyan Children with Schistosoma haematobium, Plasmodium falciparum, and other chronic parasitic co-infections. Am J Trop Med Hyg . 2015;92:945-51.

11. Maizels RM, Balic A, Gomez-Escobar N, Nair M, Taylor MD, Allen JE. Helminth parasites - Masters of regulation. Immunol. Rev. 2004. p. 89-116.

12. Arinola GO, Morenikeji OA, Akinwande KS, Alade AO, Olateru-Olagbegi O, Alabi PE, et al. Serum levels of cytokines and ige in helminth-infected nigerian pregnant women and children. Ann Glob Heal . Elsevier Inc; 2015;81:689-93.

13. Esen M, Mordmüller B, de Salazar PM, Adegnika AA, Agnandji ST, Schaumburg F, et al. Reduced antibody responses against Plasmodium falciparum vaccine candidate antigens in the presence of Trichuris trichiura. Vaccine. Elsevier Ltd; 2012;30:7621-4.

14. Ademolue TW, Aniweh Y, Kusi KA, Awandare GA. Patterns of inflammatory responses and parasite tolerance vary with malaria transmission intensity. Malar J. BioMed Central; 2017;16:145.

15. M’Bondoukwé NP, Kendjo E, Mawili-Mboumba DP, Koumba Lengongo JV, Offouga Mbouoronde C, Nkoghe D, et al. Prevalence of and risk factors for malaria, filariasis, and intestinal parasites as single infections or co-infections in different settlements of Gabon, Central Africa. Infect Dis Poverty; 
2018;7:6

16. M'Bondoukwé NP, Mawili-Mboumba DP, Mondouo Manga F, Kombila M, Bouyou-Akotet MK. Prevalence of soil-transmitted helminths and intestinal protozoa in shanty towns of Libreville, Gabon. Int J Trop Dis Heal. 2016;20:1-9.

17. Chandramathi S, Suresh K, Kuppusamy UR. Solubilized antigen of Blastocystis hominis facilitates the growth of human colorectal cancer cells, HCT116. Parasitol Res. 2010;106:941-5.

18. Long HY, Handschack A, König W, Ambrosch A. Blastocystis hominis modulates immune responses and cytokine release in colonic epithelial cells. Parasitol Res. 2001;87:1029-30.

19. Yakoob J, Abbas Z, Usman MW, Sultana A, Islam M, Awan S, et al. Cytokine changes in colonic mucosa associated with Blastocystis spp. subtypes 1 and 3 in diarrhoea-predominant irritable bowel syndrome. Parasitology. 2014;141:957-69. Available from:

https://www.cambridge.org/core/journals/parasitology/article/cytokine-changes-in-colonic-mucosa-associated-with-blastocystis-spp-subtypes-1-and-3in-diarrhoeapredominant-irritable-bowel-syndrome/3294E6ED40254E6B94A7BA9E50841F55.

20. Planche T, Krishna S, Kombila M, Engel K, Faucher JF, Kremsner PG. Comparison of methods for the rapid laboratory assessment of children with malaria. Am J Trop Med Hyg . 2001;65:599-602.

21. Ho Thi Sang, Petithory J. Techniques for the concentration of microfilariae from the Blood. Bull la Soc Pathol Exot. 1963;56:197-206.

22. Snounou G, Singh B. Nested PCR analysis of Plasmodium parasites. Methods Mol Med. 2002;72:189-203.

23. Boström S, Ibitokou S, Oesterholt M, Schmiegelow C, Persson JO, Minja D, et al. Biomarkers of Plasmodium falciparum infection during pregnancy in women living in Northeastern Tanzania. PLoS One. 2012;7.

24. Bland JM, Altman DG. Multiple significance tests: the Bonferroni method. BMJ. 1995;310:170.

25. Brutus L, Watier L, Briand V, Hanitrasoamampionona V, Razanatsoarilala H, Cot M. Parasitic co-infection: Does Ascaris lumbricoides protect against Plasmodium falciparum infection? Am J Trop Med Hyg . 2006;75:194-8.

26. Briand V, Watier L, Le Hesran J-Y, Garcia A, Cot M. Coinfection with Plasmodium falciparum and Schistosoma haematobium: protective effect of schistosomiasis on malaria in senegalese children? Am J Trop Med Hyg. 2005;72:702-7.

27. Naus CWA, Jones FM, Satti MZ, Joseph S, Riley EM, Kimani G, et al. Serological responses among individuals in areas where both schistosomiasis and malaria are endemic: cross-reactivity between Schistosoma mansoni and Plasmodium falciparum. J Infect Dis . 2003;187:1272-82.

28. Wilson NO, Bythwood T, Solomon W, Jolly PE, Yatich N, Jiang Y, et al. Elevated levels of il-10 and g-csf associated with asymptomatic malaria in pregnant women. Infect Dis Obstet Gynecol. 2010;2010.

29. Guiyedi V, Bécavin C, Herbert F, Gray J, Cazenave P-A, Kombila M, et al. Asymptomatic Plasmodium falciparum infection in children is associated with increased auto-antibody production, high IL-10 plasma levels and antibodies to merozoite surface protein 3. Malar J; $2015 ; 14: 162$.

30. de Waal Malefyt R. Interleukin 10 (IL-10) inhibits cytokine synthesis by human monocytes: an autoregulatory role of IL-10 produced by monocytes. J Exp Med . 1991;174:1209-20.

31. Kwiatkowski D. Cytokines and anti-disease immunity to malaria. Res Immunol. 1991;142:707-12.

32. Mandala WL, Msefula CL, Gondwe EN, Drayson MT, Molyneux ME, MacLennan CA. Cytokine profiles in malawian children presenting with uncomplicated malaria, severe malaria, and cerebral malaria. Clin Vaccine Immunol. 2017;24:1-11.

33. Mbengue B, Niang B, Niang MS, Varela ML, Fall B, Fall MM, et al. Inflammatory cytokine and humoral responses to Plasmodium falciparum glycosylphosphatidylinositols correlates with malaria immunity and pathogenesis. Immunity, Inflamm Dis. 2016;4:24-34.

34. Dieye Y, Mbengue B, Dagamajalu S, Fall MM, Loke MF, Nguer CM, et al. Cytokine response during non-cerebral and cerebral malaria: evidence of a failure to control inflammation as a cause of death in African adults. PeerJ . 2016;4:e1965.

35. Njama-Meya D, Kamya MR, Dorsey G. Asymptomatic parasitaemia as a risk factor for symptomatic malaria in a cohort of Ugandan children. Trop Med Int Heal. 2004;9:862-8.

36. Mawili-Mboumba DP, Bouyou-Akotet MK, Kendjo E, Nzamba J, Owono Medang M, Mourou Mbina J-R, et al. Increase in malaria prevalence and age of at risk population in different areas of Gabon. Malar J . 2013;12:1-7.

37. Akue J-P, Nkoghe D, Padilla C, Moussavou G, Moukana H, Mbou RA, et al. Epidemiology of concomitant infection due to Loa loa and Mansonella perstans in Gabon. PLoS Negl Trop Dis. 2011;5:e1329.

38. Bouyou-Akotet MK, Owono-Medang M, Moussavou Boussougou MN, Mabika-Manfoumbi M, Mintsa Nguema R, Mawili-Mboumba DP, et al. Low sensitivity of the ImmunocardSTAT ${ }^{\circledR}$ Crypto/Giardia Rapid Assay test for the detection of Giardia and Cryptosporidium in fecal samples from children living in Libreville, Central Africa. J Parasit Dis. 2015;

39. Ajjampur SSR, Tan KSW. Pathogenic mechanisms in Blastocystis spp. - Interpreting results from in vitro and in vivo studies. Parasitol Int . Elsevier B.V.; 2016;65:772-9.

40. Partida-Rodríguez O, Serrano-Vázquez A, Nieves-Ramírez ME, Moran P, Rojas L, Portillo T, et al. Human intestinal microbiota: interaction between parasites and the host immune response. Arch Med Res. 2017;

41. Frosch AE, John CC. Immunomodulation in Plasmodium falciparum malaria: experiments in nature and their conflicting implications for potential therapeutic agents. Expert Rev Anti Infect Ther. 2013;10:1343-56.

42. Dodoo D, Omer FM, Todd J, Akanmori BD, Koram K a, Riley EM. Absolute levels and ratios of proinflammatory and anti-inflammatory cytokine production in vitro predict clinical immunity to Plasmodium falciparum malaria. J Infect Dis . 2002;185:971-9. Available from:

http://www.ncbi.nlm.nih.gov/pubmed/11920322

Page $9 / 13$ 
43. Perret JL, Duong TH, Kombila M, Owono M, Nguemby-Mbina C. [Results of a systematic search for Plasmodium in internal medicine in Gabon]. Bull Soc Pathol Exot . 1991;84:323-9.

44. Nkoghe D, Akue J-P, Gonzalez J-P, Leroy EM. Prevalence of Plasmodium falciparum infection in asymptomatic rural Gabonese populations. Malar J. BioMed Central Ltd; 2011;10:33. Available from: http://www.malariajournal.com/content/10/1/33

45. Degarege A, Erko B. Epidemiology of Plasmodium and Helminth Coinfection and Possible Reasons for Heterogeneity. Biomed Res Int. Hindawi Publishing Corporation; 2016;2016:1-6. Available from: http://www.hindawi.com/journals/bmri/2016/3083568/

46. Figueiredo CA, Barreto ML, Rodrigues LC, Cooper PJ, Silva NB, Amorim LD, et al. Chronic intestinal helminth infections are associated with immune hyporesponsiveness and induction of a regulatory network. Infect Immun. 2010;78:3160-7.

47. Winkler S, Willheim M, Baier K, Aichelburg A, Kremsner PG, Graninger W. Increased frequency of TH2-type cytokine-producing T cells in microfilaremic loiasis. Am J Trop Med Hyg. 1999;60:680-6.

48. Bouyou-Akotet MK, Mawili-Mboumba DP, Kendjo E, Eyang Ekouma A, Abdou Raouf O, Engohang Allogho E, et al. Complicated malaria and other severe febrile illness in a pediatric ward in Libreville, Gabon. BMC Infectious Diseases; 2012;12:216.

\section{Tables}

Table 1: Distribution of the groups with different parasitic profile according to sex, age and location

\begin{tabular}{|c|c|c|c|c|c|c|c|c|c|c|c|c|c|c|c|c|c|c|}
\hline & Total & $\begin{array}{l}\text { No } \\
\text { para } \\
(\mathrm{n}=\end{array}$ & $\begin{array}{l}\text { sites } \\
\text { 35) }\end{array}$ & $\begin{array}{l}P . \\
\text { falc } \\
\text { only } \\
(\mathrm{n}=\end{array}$ & $\begin{array}{l}\text { parum } \\
\text {;0) }\end{array}$ & $\begin{array}{l}\text { Fila } \\
(\mathrm{n}=\end{array}$ & & $\begin{array}{l}\text { STF } \\
(n=\end{array}$ & & $\begin{array}{l}\text { Inte } \\
\text { prot } \\
\text { only } \\
(\mathrm{n}=\end{array}$ & $\begin{array}{l}\text { stinal } \\
\text { ozoa }\end{array}$ & $\begin{array}{l}\mathrm{Pla} \\
\mathrm{Co}- \\
(\mathrm{n}=\end{array}$ & $\begin{array}{l}\text { ectium/filariae } \\
\text { ection }\end{array}$ & & $\begin{array}{l}\text { modium/STH } \\
\text { fection }(n=7)\end{array}$ & $\begin{array}{l}\text { Plas } \\
\text { protc } \\
(\mathrm{n}=\end{array}$ & $\begin{array}{l}\text { ium/intestinal } \\
\text { co-infection }\end{array}$ & $P$ \\
\hline & $\mathbf{N}$ & $\mathbf{n}$ & $\%$ & $\mathbf{n}$ & $\%$ & $\mathbf{n}$ & $\%$ & $\mathbf{n}$ & $\%$ & $\mathbf{n}$ & $\%$ & $\mathbf{n}$ & $\%$ & $\mathbf{n}$ & $\%$ & $\mathbf{n}$ & $\%$ & \\
\hline Male sex & 109 & 17 & 48.6 & 24 & 48.0 & 21 & 47.7 & 11 & 26.8 & 20 & 51.3 & 3 & 42.9 & 4 & 57.1 & 9 & 52.9 & 0.4 \\
\hline $\begin{array}{l}\text { Age } \\
\text { groups }\end{array}$ & 224 & 35 & & 49 & & 43 & & 34 & & 39 & & 7 & & 5 & & 17 & & $<0.000$ \\
\hline $\begin{array}{l}0-4 \text { years } \\
\text { old }\end{array}$ & 47 & 13 & 37.2 & 16 & 32.6 & 0 & 0.0 & 4 & 11.8 & 9 & 23.1 & 0 & 0.0 & 3 & 50.0 & 2 & 11.8 & \\
\hline $\begin{array}{l}5-15 \\
\text { years old }\end{array}$ & 47 & 6 & 17.1 & 19 & 38.8 & 1 & 2.3 & 6 & 17.7 & 11 & 28.2 & 0 & 0.0 & 1 & 16.7 & 9 & 52.9 & \\
\hline $\begin{array}{l}>15 \text { years } \\
\text { old }\end{array}$ & 130 & 16 & 45.7 & 14 & 28.6 & 42 & 97.7 & 24 & 70.5 & 19 & 48.7 & 7 & 100.0 & 2 & 33.3 & 6 & 35.3 & \\
\hline Location & 208 & 30 & & 49 & & 41 & & 37 & & 27 & & 7 & & 7 & & 10 & & $<0.000$ \\
\hline $\begin{array}{l}\text { Urbanized } \\
\text { area }\end{array}$ & 36 & 12 & 40.0 & 15 & 30.6 & 0 & 0.0 & 0 & 0.0 & 6 & 22.2 & 0 & 0.0 & 0 & 0.0 & 3 & 30.0 & \\
\hline $\begin{array}{l}\text { Rural } \\
\text { area }\end{array}$ & 172 & 18 & 60.0 & 34 & 69.4 & 41 & 100.0 & 37 & 100.0 & 21 & 77.8 & 7 & 100.0 & 7 & 100.0 & 7 & 70.0 & \\
\hline
\end{tabular}

p-values were obtained using the Fisher's exact test.

Table 2: Median level of pro- and anti-inflammatory cytokines according to parasitic profile

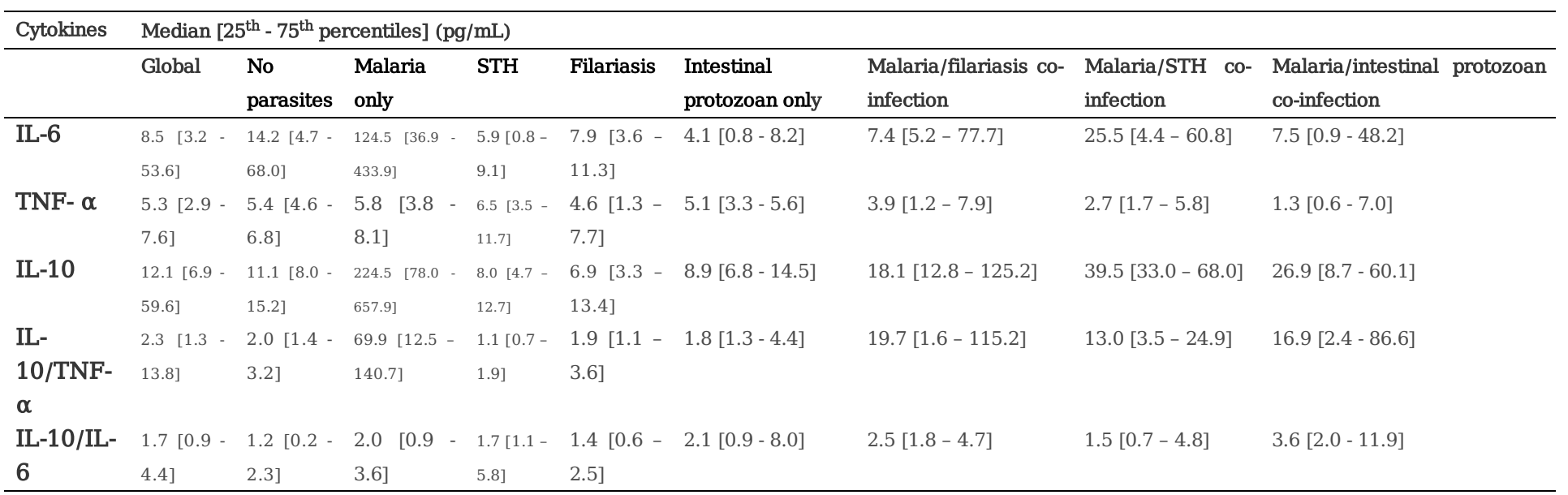

Table 2 shows median levels of cytokines and ratios in different parasitic groups. $P$-values were obtained with the Kruskal-Wallis $\mathrm{H}$-test. IL-6: $\chi^{2}=65.725, d f=7, P<0.0001 ;$ TNF- $\alpha: \chi^{2}=11.107, d f=7, P=0.09 ;$ IL-10 : $\chi^{2}=87.714, d f=7, P<0.0001 ; \mathrm{IL}-10 / \mathrm{TNF}-\boldsymbol{\alpha}: \chi^{2}=81.337$, $d f=7, P<0.0001 ;$ IL-10/IL-6 $: \chi^{2}=14.781, d f=7, P=0.04$. 
Table 3: median level of cytokines according to single parasitism and age groups

\begin{tabular}{|c|c|c|c|c|}
\hline \multicolumn{5}{|c|}{ Median [25 th $-75^{\text {th }}$ interquartiles] } \\
\hline Groups & & $0-4$ years old & 5-15 years old & $>15$ years old \\
\hline No parasites & $\begin{array}{l}\text { IL-6 } \\
\text { TNF- } \alpha \\
\text { IL-10 } \\
\text { IL-10/TNF- } \alpha \\
\text { IL-10/IL-6 }\end{array}$ & $\begin{array}{l}40.7[12.2-124.1] \\
5.3[4.6-5.5] \\
13.1[11.1-18.5] \\
2.4[2.2-3.7] \\
0.4[0.1-1.4]\end{array}$ & $\begin{array}{l}30.1[0.8-99.8] \\
5.2[4.5-6.5] \\
10.5[6.9-223.8] \\
2.3[1.2-25.4] \\
5.0[1.3-8.6]\end{array}$ & $\begin{array}{l}8.9[4.1-39.4] \\
6.2[4.2-8.0] \\
9.6[7.0-12.9] \\
1.6[1.3-1.9] \\
1.3[0.6-3.2]\end{array}$ \\
\hline Malaria only & $\begin{array}{l}\text { IL-6 } \\
\text { TNF- } \alpha \\
\text { IL-10 } \\
\text { IL-10/TNF- } \alpha \\
\text { IL-10/IL-6 }\end{array}$ & $\begin{array}{l}198.1[64.4-515.7] \\
5.0[4.4-7.8] \\
180.1[119.3-649.0] \\
60.9[16.0-111.1] \\
1.4[0.7-2.8]\end{array}$ & $\begin{array}{l}137.8[43.8-968.3] \\
6.4[5.1-8.1] \\
424.4[59.5-755.4] \\
75.1[8.0-132.5] \\
2.1[0.4-3.5]\end{array}$ & $\begin{array}{l}67.1[23.0-213.1] \\
5.6[0.6-8.1] \\
196.2[38.4-820.4] \\
80.7[6.5-486.3] \\
2.1[1.0-5.5]\end{array}$ \\
\hline Filariasis & $\begin{array}{l}\text { IL-6 } \\
\text { TNF- } \alpha \\
\text { IL-10 } \\
\text { IL-10/TNF- } \alpha \\
\text { IL-10/IL-6 }\end{array}$ & $\begin{array}{l}- \\
- \\
- \\
- \\
-\end{array}$ & $\begin{array}{l}11.2 \\
9.3 \\
32.1 \\
3.4 \\
2.9\end{array}$ & $\begin{array}{l}7.6[3.3-11.0] \\
4.6[1.2-7.7] \\
6.9[3.2-12.9] \\
2.0[1.1-3.4] \\
1.4[0.6-2.5]\end{array}$ \\
\hline STH & $\begin{array}{l}\text { IL-6 } \\
\text { TNF- } \alpha \\
\text { IL-10 } \\
\text { IL-10/TNF- } \alpha \\
\text { IL-10/IL-6 }\end{array}$ & $\begin{array}{l}1.0[0.8-4.8] \\
7.2[4.6-10.9] \\
7.1[6.0-10.7] \\
1.0[0.8-1.5] \\
7.1[4.1-8.8]\end{array}$ & $\begin{array}{l}5.0[0.8-11.4] \\
3.4[2.0-3.8] \\
4.7[3.4-15.2] \\
2.0[0.9-4.7] \\
2.9[0.2-5.8]\end{array}$ & $\begin{array}{l}2.6[0.8-8.0] \\
6.4[3.4-10.9] \\
8.3[4.1-12.9] \\
1.1[0.7-2.0] \\
2.1[1.1-5.5]\end{array}$ \\
\hline Intestinal protozoa only & $\begin{array}{l}\text { IL-6 } \\
\text { TNF- } \alpha \\
\text { IL-10 } \\
\text { IL-10/TNF- } \alpha \\
\text { IL-10/IL-6 }\end{array}$ & $\begin{array}{l}13.9[3.3-119.5] \\
4.3[3.1-5.1] \\
16.7[9.7-54.6] \\
4.8[2.5-13.0] \\
2.4[1.0-5.4]\end{array}$ & $\begin{array}{l}5.0[0.8-7.1] \\
5.3[4.6-6.3] \\
8.7[7.1-13.1] \\
1.6[1.3-2.8] \\
2.0[1.2-8.8]\end{array}$ & $\begin{array}{l}3.1[0.8-6.0] \\
5.1[0.9-5.7] \\
7.6[5.7-223.8] \\
1.5[1.2-2.1] \\
2.1[0.7-7.9]\end{array}$ \\
\hline
\end{tabular}

\section{Figures}

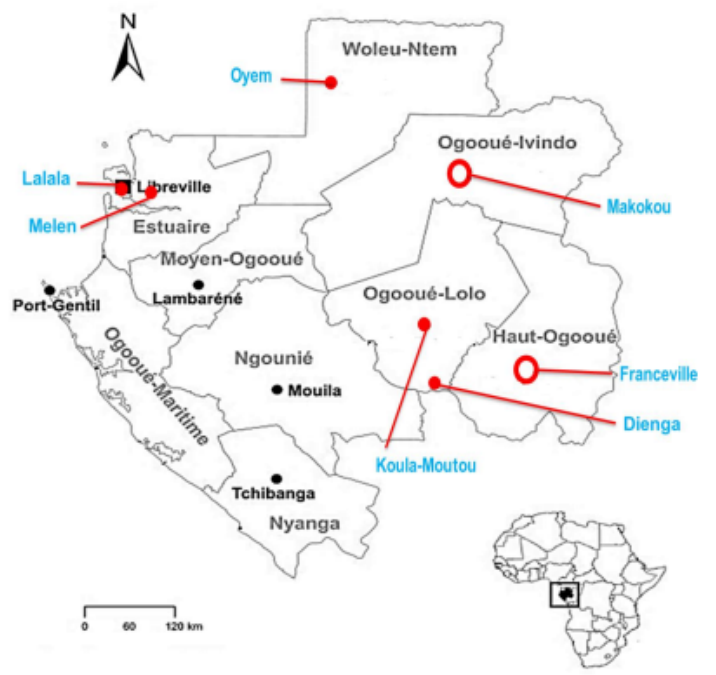

Figure 1

Study sites where samples were collected. Red circles represent many villages around the site and red full stop, one site. 


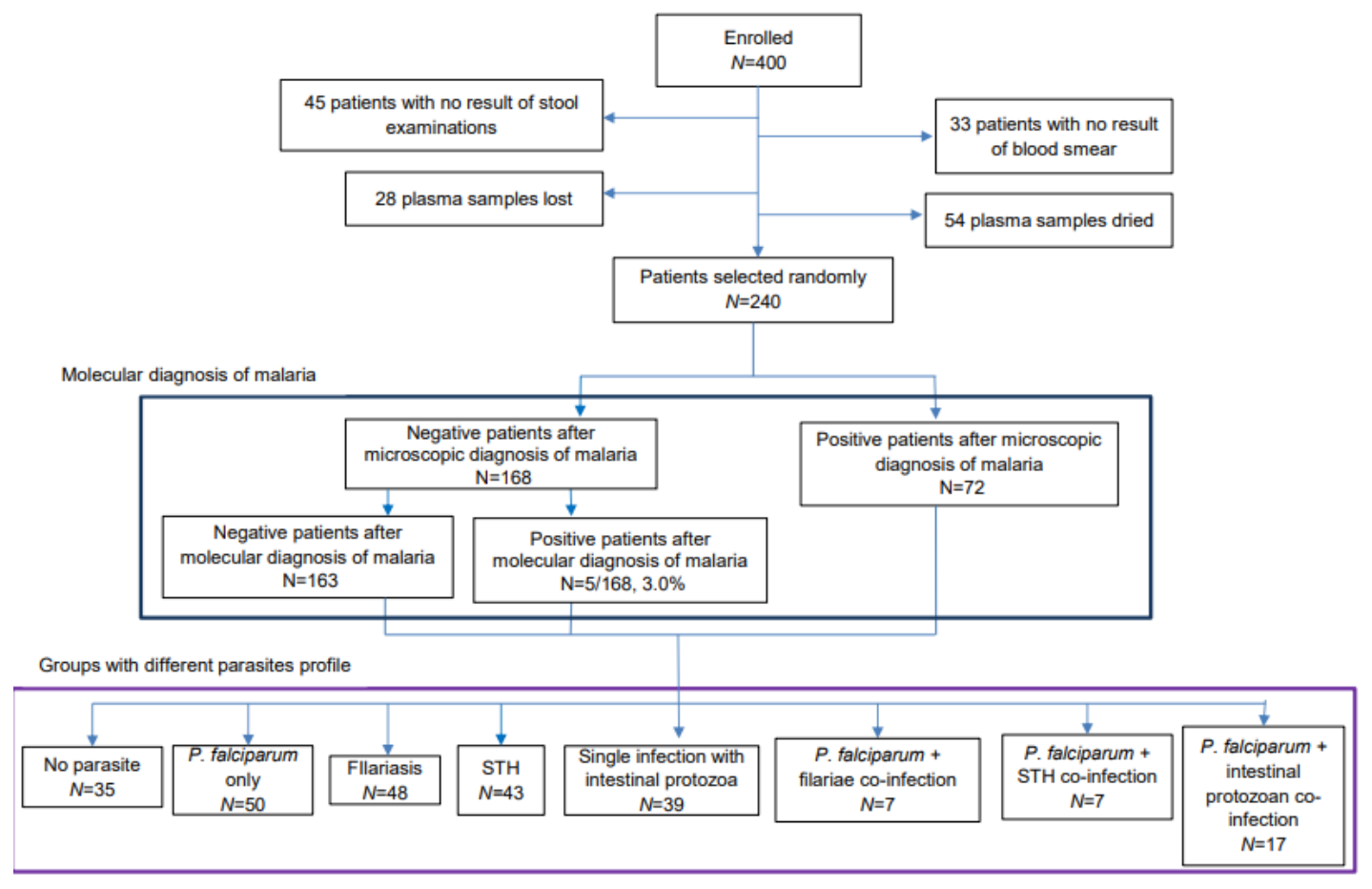

\section{Figure 2}

represents a flow chart ranging to the enrolled population to absolute frequencies in different groups with different parasite profile.
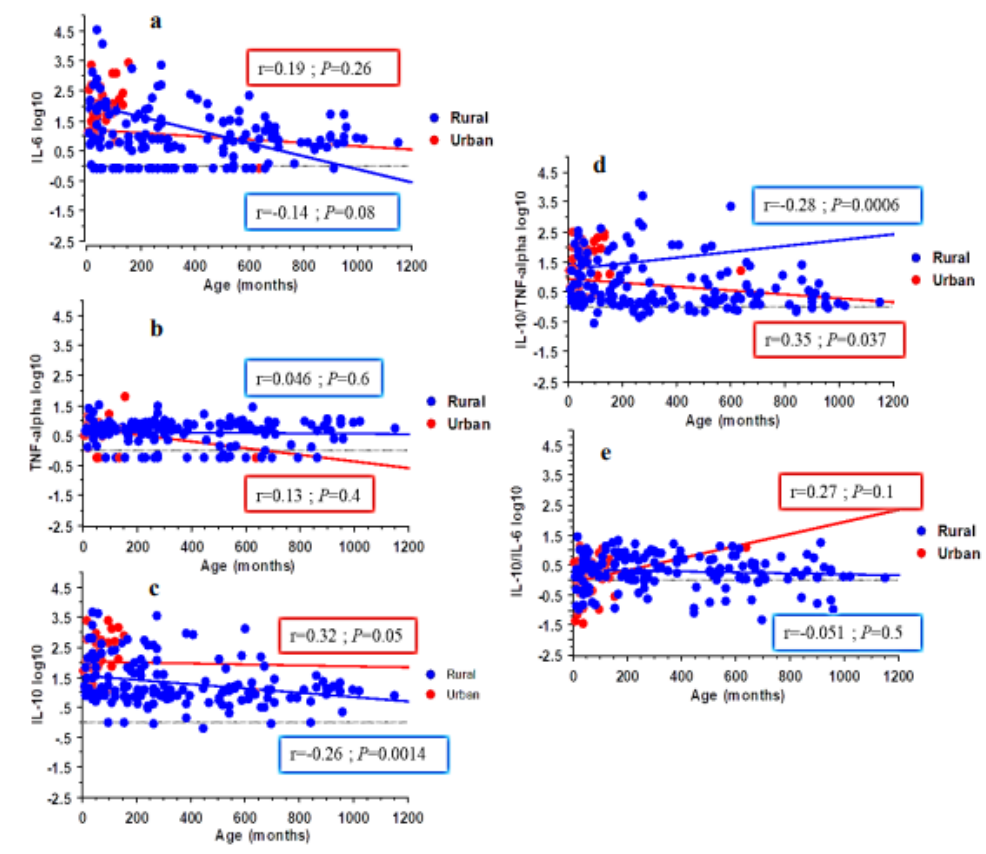

Figure 3

Cytokines profiles stratified by areas according to age in months. Correlations between IL-6 (a) TNF-a (b) IL-10 (c) IL-10/TNF-a (d) IL-10/IL-6 (e) levels and the age of participants in months. decreased when age increased in rural area. IL-10/TNF-alpha ratio increased with age in rural are but decreased in urban area. 


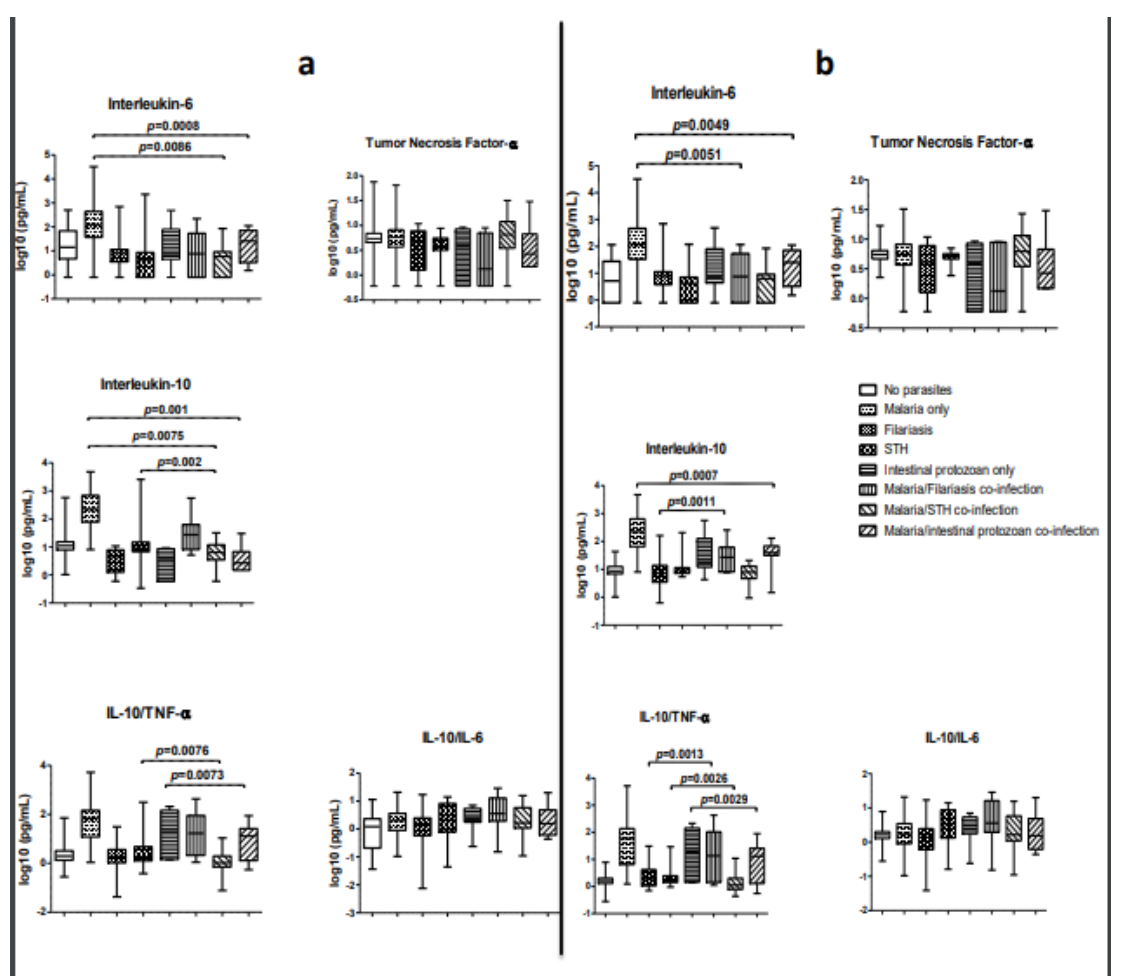

\section{Figure 4}

Box plot displaying IL-6, TNF-a and IL-10 cytokine production and IL-10/TNF-a, IL-10/IL-6 median ratios according to different parasitic profile in the entire population (a) and in rural area (b). Mann-Whitney U-test was carried to pairwise comparisons. Values used for the graphical representation were logtransformed.

\section{Supplementary Files}

This is a list of supplementary files associated with this preprint. Click to download.

- Additionalfile1tables1.docx

- graphicalabstract.pdf 